\title{
Unusual Growth and Hydration Characteristics of Oil Solubilized Micelles in Aqueous Pluronic System
}

\section{R. Ganguly, ${ }^{1}$ S. Kumar, ${ }^{2}$ S. Nath, ${ }^{3}$ M. Basu ${ }^{1}$ and V.K. Aswal ${ }^{2}$}

${ }^{1}$ Chemistry Division, Bhabha Atomic Research Centre, Mumbai-400085, India

${ }^{2}$ Solid State Physics Division, Bhabha Atomic Research Centre, Mumbai-400085, India

${ }^{3}$ Radiation \& Photochemistry Division, Bhabha Atomic Research Centre, Mumbai-400085, India

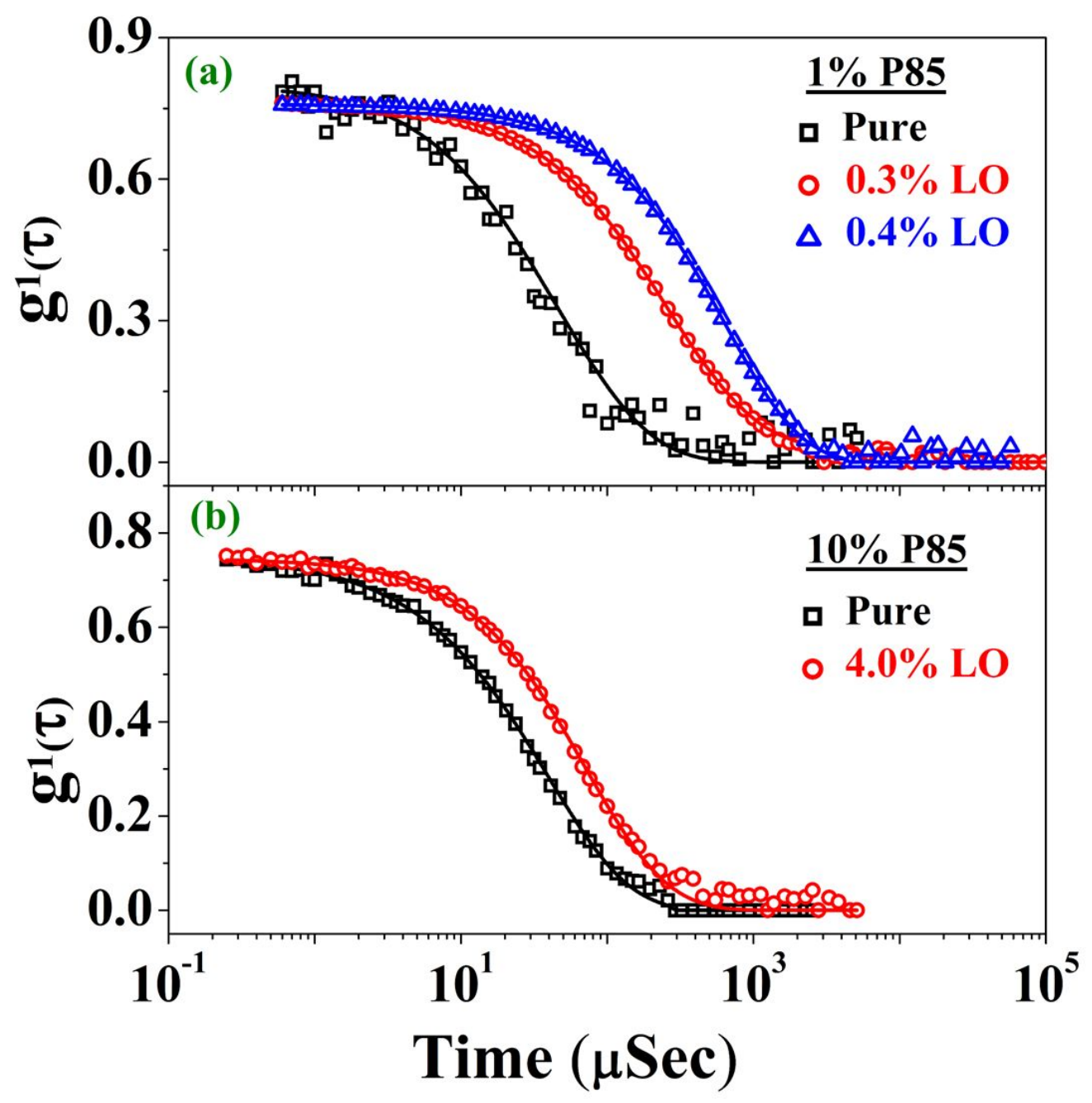

Figure S1. Correlation function diagrams recorded at $130^{\circ}$ scattering angle and $30^{\circ} \mathrm{C}$ for (a) $1 \mathrm{wt}$. $\%$ and (b) 10 wt. \% P85 solutions at different lavender oil (LO) concentrations. Solid lines in the plots represent fit to the data by the method of CONTIN. 


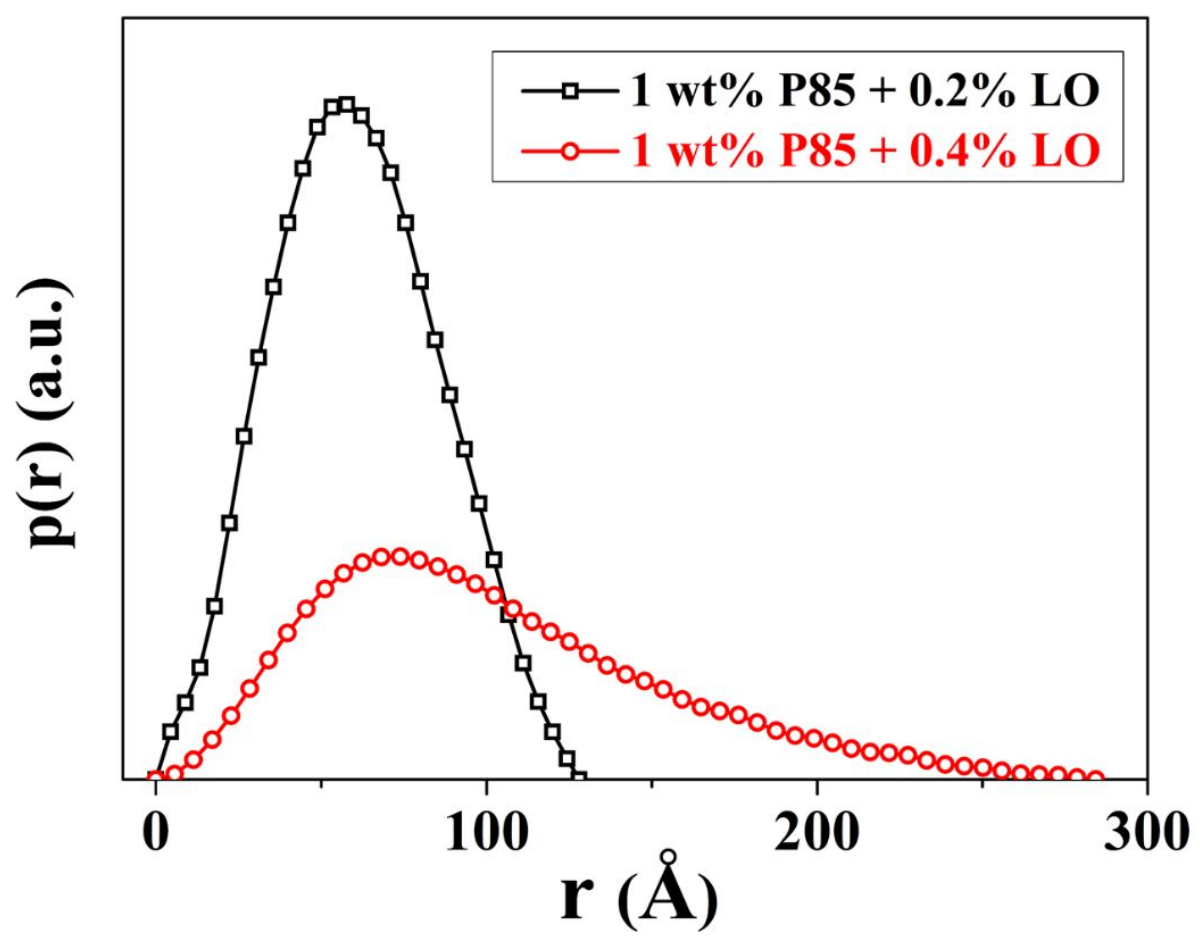

Figure S2. Pair distribution function plots obtained from SANS data for 1\% P85 solutions with varying LO concentrations.

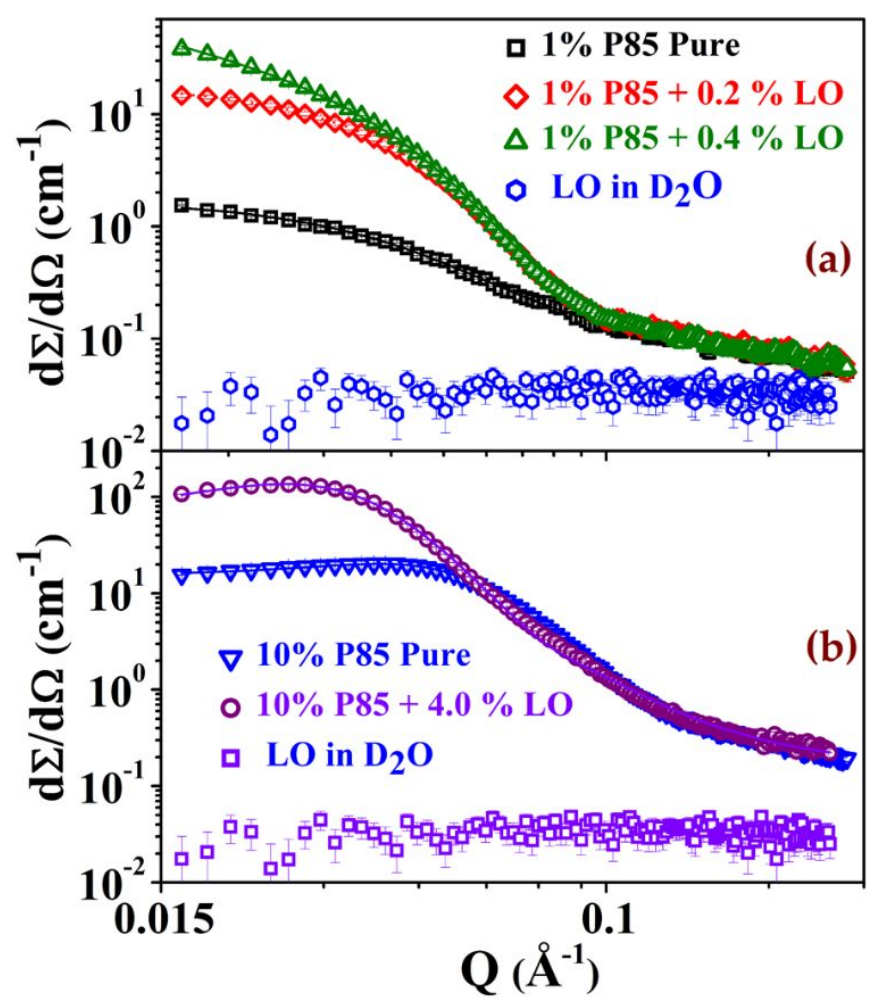

Figure S3. SANS patterns of pure and LO solubilized (a) 1\%, (b) 10\% P85 solutions. The solid lines represent fit to the data. 


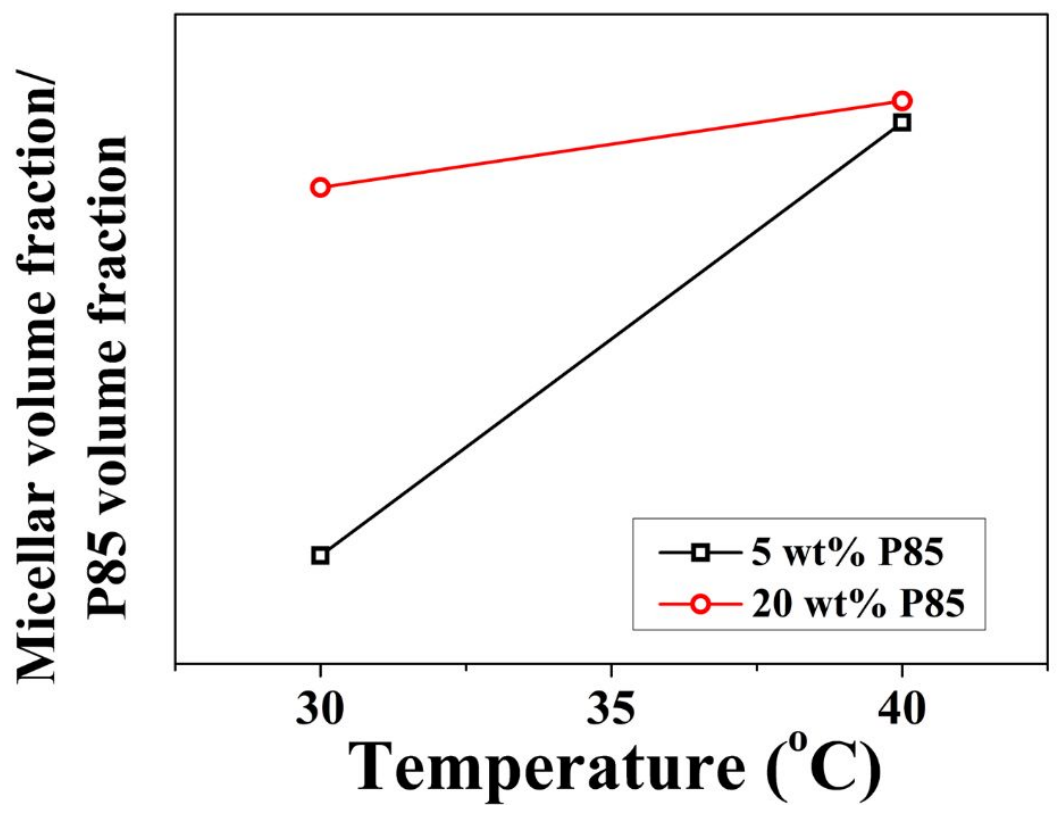

Figure S4. Ratio of micellar volume fraction to P85 volume fraction for 5\% and 20\% P85 solutions at $30^{\circ} \mathrm{C}$ and $40^{\circ} \mathrm{C}$.

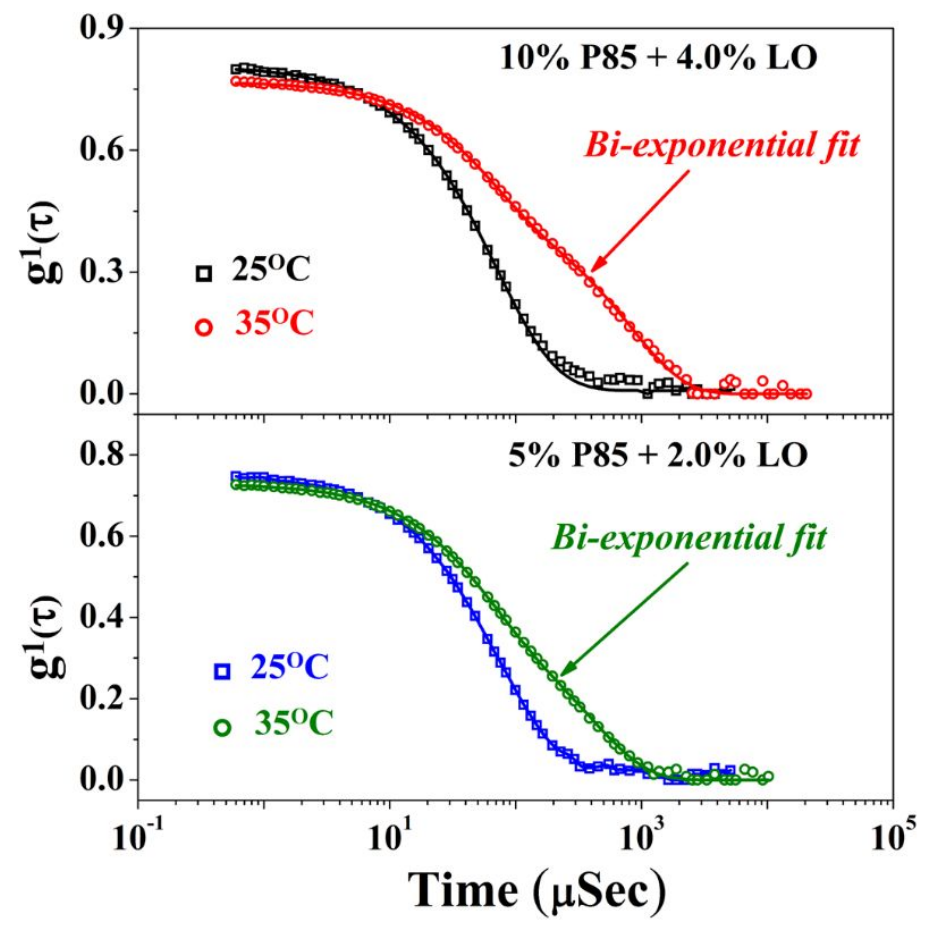

Figure S5. Correlation function diagrams recorded at $130^{\circ}$ scattering angle for LO solubilized P85 solutions at different temperatures. Solid lines in the plots represent fit to the data by the method of CONTIN and biexponential equation. 
The form of the bi-exponential equation used for fitting of the data in figure S5 is:

$$
A * \exp \left(-\Gamma^{1 *} t\right)+B * \exp \left(-\Gamma^{2 * t}\right)
$$

where $\mathrm{A}$ and $\mathrm{B}$ are relative amplitudes for the scattering species of two different sizes; $\Gamma^{1}$ and $\Gamma^{2}$ represent the decay rates of the corresponding contributions in the correlation function. The fitted values obtained for LO solubilized $10 \%$ and $5 \% \mathrm{P} 85$ solutions at $35^{\circ} \mathrm{C}$ are shown in table $\mathrm{S} 1$.

Table S1: Parameters obtained from fitting of the correlation function plots for LO solubilized 10\% and $5 \% \mathrm{P} 85$ solutions recorded at $35^{\circ} \mathrm{C}$ by double exponential equation as mentioned in the text above. The ratio of the concentrations of pluronics and LO kept constant in the two samples.

\begin{tabular}{|c|c|c|c|c|}
\hline Sample & $\mathrm{A}$ & $\Gamma^{1}\left(\mathrm{Sec}^{-1}\right)$ & $\mathrm{B}$ & $\Gamma^{2}\left(\mathrm{Sec}^{-1}\right)$ \\
\hline $10 \% \mathrm{P} 85+4.0 \% \mathrm{LO}$ & 0.32 & 0.0169 & 0.45 & 0.0012 \\
\hline $05 \%$ P85 + 2.0\% LO & 0.33 & 0.0188 & 0.40 & 0.0024 \\
\hline
\end{tabular}

